\title{
Adult Penile Carcinoma
}

National Cancer Institute

\section{Source}

National Cancer Institute. Adult Penile Carcinoma. NCI Thesaurus. Code C118820.

A carcinoma of the penis that occurs during adulthood. 